\title{
Training the next generation of cardiac surgeons: is it time to redesign the case requirements?
}

\author{
George Tolis Jr. ${ }^{1}$ (D) \\ Received: 30 April 2020 / Accepted: 13 May 2020 / Published online: 22 June 2020 \\ (C) Indian Association of Cardiovascular-Thoracic Surgeons 2020
}

Cardiac surgery has undergone a number of changes in the past three decades. New procedures have been introduced and popularized, some others have been partially replaced by "less invasive" or "endovascular" alternatives, while many have remained the same, but have been subjected to significant changes in volume and are being offered to sicker and older patients.

The Thoracic Surgery Licensing Boards of several countries, including the American Board of Thoracic Surgery (ABTS), have attempted to keep up with these changes, mostly by modifying the case number requirements of established procedures, while at the same time creating new case requirement numbers for procedures that have only been recently introduced into cardiac surgery practices. As a result, we have seen the requirements of pacemaker insertions vanish altogether ( 5 generator removal procedures satisfy the ABTS "pacemaker" requirements). On the other hand, case requirement numbers for transcatheter valves have been recently introduced, with the ABTS requiring 5 transcatheter aortic valve replacements (TAVR) as surgeon and 10 as a first assistant. Clearly, an attempt is constantly being made to modify the licensing requirements based on the changing face of cardiac surgery in order to make sure that the graduating fellows are prepared to enter an active clinical practice.

Despite the ongoing changes in the case type and number requirements, no changes have taken place in the core metric of all these procedures, which is none other than the number of cases performed. When a simpler operation is being evaluated, such as a nevus removal in a dermatology outpatient practice or a diagnostic cardiac catheterization, the actual number of

George Tolis, Jr.

gtolis@mgh.harvard.edu

1 Division of Cardiac Surgery, Massachusetts General Hospital, Harvard Medical School, 55 Fruit Street, Boston, MA 02114, USA procedures involved is likely to accurately represent a trainee's experience. However, with a more complicated procedure, the range of actual trainee involvement can vary significantly in cases where the trainees have listed themselves as the operating surgeon and have claimed credit for the procedure.

It is a well known, yet not widely discussed fact, that in many programs, residents have to "count" cases where their involvement was minimal in order to satisfy the graduating requirements. In the eyes of the licensing board, a case where a trainee simply opened the chest and performed two half anastomoses from the assistant's side of the table counts the same as a case in another program, where a trainee did the entire operation "skin-to-skin." Other trainees with a more stringent approach to case documentation may not count a case, unless they have performed the entire procedure themselves with minimal presence or input from the attending physician. Even though the correct approach to one's education may lie in between these two extreme practices, there is little doubt that the current case monitoring system is subject to the trainee's individual creative interpretation $[1,2]$. A cardiac operation is often described as a series of multiple small operative tasks, which are pieced together to form a big operation. Let us think of a coronary bypass procedure as an example: the surgeon opens the bone and obtains hemostasis; harvests one or two internal mammary arteries (IMA); cannulates the patient (if they will be performing the operation "on pump"); performs a series of proximal and distal anastomoses; and separates from cardiopulmonary bypass, decannulates, obtains hemostasis, and closes the wound. These steps are discreet operative tasks which are not interdependent, yet their organized sequential execution leads to a single complex operation.

The ABTS myocardial revascularization requirement is 80 "cases" for a cardiac track thoracic surgery resident. Clearly, if a trainee has performed 80 coronary bypass operations "skinto-skin," they should be well prepared to enter a rigorous clinical practice upon graduation from their program. 
However, there are very few-if any - programs that offer this kind of experience. Most trainees perform a part of the operation and then log the operation as a coronary artery bypass graft (CABG). One down, 79 to go...

It would make much more sense to relax the number of actual cases and create new category requirements for individual parts of the operation. For example, assuming that all 80 of these coronary bypass procedures will require an IMA to be harvested, the board could create a new category of 80 left or right IMA graft harvests that need to be performed prior to graduation. Similarly, since most valve and CABG procedures will require a sternotomy and cannulation procedure, the board can set 100 sternotomies/cannulations as the graduation requirement.

When it comes to individual coronary anastomoses and assuming that the average bypass procedure includes approximately 3 distal anastomoses, a requirement of 150-200 distal and 100-150 proximal anastomoses can be established as well. These anastomoses should be performed from the surgeon's side of the table and should be complete, rather than "my forehands and your forehands" kind of arrangement. This will enable a more accurate representation of the trainee's actual experience. Additionally, it will contribute toward the trainee's achievement of the number requirements for graduation, since individual parts, rather than complete cases, will be added into the case log.

When it comes to valve procedures, the requirement should be that the trainee places and ties down at least $80 \%$ of the valve or ring sutures. The sutures should be placed from the surgeon's side of the table and should be followed by closure of the aorta or left/right atrium, depending on the valve that is being addressed. This will guarantee that a meaningful resident experience is being recorded while giving the attending physician the flexibility to perform themselves a technically challenging tissue bite or knot tying without compromising the care delivered to the patient. A total of 20 aortic valve replacements and 20 mitral replacements/repairs recorded in this fashion should be enough to guarantee that the trainee is prepared to enter an active clinical practice.

Similar requirements can also be drafted for less common procedures such as ascending aortic aneurysm and root replacements and arch replacements. Obviously, the case number requirements will be lower than what they are for the more common procedures.

Having said that, it is still important for residents to have completed entire cases in order to display their ability to "put the whole thing together." However, if they have satisfied the individual step requirements, as recommended by their licensing board, the number of "total cases" does not have to exceed 20 cases or so and it can include any kind of case (coronary, valve etc.), as long as the resident has completed the vast majority of the case from the surgeon's side of the table and has displayed their ability to conduct the operation with minimal attending physician guidance.

These recommendations may be novel in the world of surgical education, but they are part of the established way of training and certification in other fields that involve teaching of a complicated task, which is made up of a series of smaller steps. In the aviation industry, for example, the number of flights is only one of many metrics that is monitored during training and subsequent recertification. The number of takeoffs, landings (visual or instrument), and carrier landing for naval aviators are individual metrics that are also taken into account in addition to the actual number of flights and hours of flying.

In our modern era of older and sicker patients, publicly available quality metrics, patient demands, and competing less invasive technologies, making sure that the future generations of heart surgeons not only remain current with recent advances in our field, but also stay comfortable with traditional surgical approaches, are of utmost importance. The current assessment system which uses the "number of cases" as the only metric leading to certification is out of date and in need of significant modifications. It is now time to redraft the graduation requirements for our trainees using a system that accurately reflects the operative experience they obtained during their training and is not susceptible to the creative interpretation of the trainee or their supervising program director.

\section{Compliance with ethical standards}

Conflicts on Interest Nil declared.

\section{References}

1. Tolis G Jr, Spencer PJ, Bloom JP, et al. Teaching operative cardiac surgery in the era of increasing patient complexity: can it still be done? J Thorac Cardiovasc Surg. 2018;155:2058-65.

2. Tolis G. Cardiac surgical operative training: a disincentivized necessity. Eur J Cardiothorac Surg. 2019;55:173-8.

Publisher's note Springer Nature remains neutral with regard to jurisdictional claims in published maps and institutional affiliations. 